\title{
Preparative Isolation of Peroxisomes from Liver and Kidney Using Metrizamide Density Gradient Centrifugation in a Vertical Rotor
}

\author{
AMIYA K. HAJRA AND DOROTHY WU \\ Neuroscience Laboratory, Mental Health Research Institute, and Department of Biological Chemistry, \\ University of Michigan, 1103 E. Huron, Ann Arbor, Michigan 48109
}

Received December 26, 1984

\begin{abstract}
A method for the preparative isolation of peroxisomes from the livers of rat, guinea pig, and mouse, and also from rat kidney is described. The light mitochondrial fraction, i.e., particles sedimenting between 33,000 and $250,000 \mathrm{~g}$-min, or the postnuclear supernatant of liver or kidney, is subjected to a $20-50 \%$ Metrizamide density gradient ultracentrifugation in a vertical rotor. After centrifugation, the peroxisomes (marker enzyme catalase and dihydroxyacetone phosphate acyltransferase) sedimented as a band near the bottom of the tube $(\rho=1.22 \mathrm{~g} / \mathrm{ml})$. From the distribution of different marker enzymes and also from the morphometric examinations, it was demonstrated that the isolated peroxisomes are not contaminated with lysosomes, mitochondria, or microsomes. 1985 Academic Press, Inc.

KEY WORDS: subcellular fractionation; peroxisomes; density gradient ultracentrifugation; Metrizamide.
\end{abstract}

Peroxisomes were first described by de Duve and co-workers as hepatic and renal subcellular organelles $(0.5-1 \mu \mathrm{m}$ diameter $)$ containing catalase and $\mathrm{H}_{2} \mathrm{O}_{2}$-producing oxidases $(1,2)$. Novikoff and co-workers described the widespread occurrence of somewhat smaller $(0.1-0.5 \mu \mathrm{m})$ catalase-containing particles, designated as microperoxisomes, in a variety of different tissues $(3,4)$. Because of their similarities in size and density with other organelles, especially with lysosomes in liver and kidney, the peroxisomes are difficult to isolate from tissue homogenates by either differential or density gradient centrifugations $(1,5)$. The most widely used method to isolate peroxisomes from liver is that introduced by Leighton et al. (5), where peroxisomes are separated from lysosomes by centrifugation in a sucrose density gradient after first lowering the density of liver lysosomes by pretreating the animals with Triton WR1339. We have successfully used that method to show that some lipid-metabolizing enzymes such as dihydroxyacetone phosphate
(DHAP) ${ }^{1}$ acyltransferase are present in liver peroxisomes (6). This method, however, cannot be used to isolate peroxisomes from other organs, such as kidney. In addition, the treatment of animals with the detergent Triton WR-1339 has been shown to alter the activities of different peroxisomal enzymes (7). A number of other density gradient centrifugation methods using either sucrose (8-10), Metrizamide (2-[3-acetamido-5- $\mathrm{N}$ methylacetamido-2,4,6-triodobenzamido[-2deoxy-D-glucose) $(11,12)$, or Percoll $(13-15)$ solutions have been described for the isolation of peroxisomes from normal animals. Most of these methods, however, yield peroxisomes which are contaminated with varying amounts of other organelles. In 1981 we described a method for isolating peroxisomes from rat and guinea pig livers using Metrizamide density gradient centrifugation in a vertical rotor and presented preliminary data

\footnotetext{
${ }^{1}$ Abbreviations used: DHAP, dihydroxyacetone phosphate; GP, sn-glycerol-3-phosphate.
} 
which indicate that the isolated peroxisomes are uncontaminated with other organelles (16). We also successfully used this method to isolate peroxisomes from kidney. The details of the method and the results are presented here.

\section{MATERIALS}

Palmitoyl CoA, DHAP, cytochrome $c$, $D$-glucose-6-phosphate, $\beta$-glycerophosphate, succinate, and isocitrate were obtained from Sigma Chemical Company (St. Louis, Mo.). sn-Glycerol-3-phosphate (GP) was from CalBiochem-Behring. Analytical-grade Metrizamide $^{2}$ (Nyegaard \& Co.) was obtained from Accurate Chemical \& Scientific Research Corporation (Westbury, N. Y.). $\left[{ }^{32} \mathrm{P}\right]-$ DHAP and ${ }^{32}$ PJGP were prepared by the enzymatic phosphorylation of dihydroxyacetone and glycerol, respectively, with $[\gamma-$ ${ }^{32}$ P]ATP, as described previously (17). [1$\left.{ }^{14} \mathrm{C}\right]$ Palmitoyl $\mathrm{CoA}$ was chemically prepared from $\left[1-{ }^{14} \mathrm{C}\right]$ palmitic acid and $\mathrm{CoASH}$ as described by Bishop and Hajra (18). Other materials were the same as described before $(6,17)$.

\section{METHODS}

Preparation of liver and kidney subcellular fractions. The subcellular fractions were prepared by differential centrifugation of the organ homogenates essentially by the method of de Duve and co-workers (19). The animals were lightly anesthetized with diethyl ether and decapitated, and the liver or kidneys were quickly extracted and chilled on ice. The organs were homogenized (one-stroke) in $3 \mathrm{vol}(\mathrm{ml} / \mathrm{g})$ of ice-cold $0.25 \mathrm{M}$ sucrose containing Tris- $\mathrm{HCl}$ buffer $(\mathrm{pH} 7.4,10 \mathrm{mM})$ and EDTA $(1.0 \mathrm{mM}$ in a glass homogenizer with a Teflon pestle (Arthur Thomas Co.) at $1500 \mathrm{rpm}$ as described before $(19,20)$. The homogenate was filtered through cheesecloth and then centrifuged at $4^{\circ} \mathrm{C}$ for $10 \mathrm{~min}$ at

\footnotetext{
${ }^{2}$ The analytical-grade Metrizamide, not the centrifugation grade (Metrizamide-C), must be used for the isolation of peroxisomes uncontaminated with lysosomes and mitochondria.
}

$800 \mathrm{~g}$ (Sorvall RC2B centrifuge). The supernatant was saved and the residue was rehomogenized as above in $3 \mathrm{vol}(\mathrm{ml} / \mathrm{g}$ of original tissue) of the cold sucrose-tris-EDTA solution and recentrifuged at $800 \mathrm{~g}$ for $10 \mathrm{~min}$. This second supernatant was combined with the first supernatant and when required this combined (1:6 homogenate) postnuclear supernatant (S-1) was used for the isolation of peroxisomes by the Metrizamide density gradient centrifugation (see later). For the isolation of the light mitochondrial or " $L$ " fraction of de Duve et al. (19), the residue obtained after the second centrifugation was rehomogenized as before in $3 \mathrm{vol}$ of sucroseTris-EDTA and recentrifuged $(800 \mathrm{~g}, 10 \mathrm{~min})$. The residue was discarded and the supernatant was combined with the other supernatants. " $L$ " fraction was isolated from this combined postnuclear supernatant (1:9 homogenate) by differential centrifugation (fraction sedimenting between 33,000 and $250,000 \mathrm{~g}$ - $\mathrm{min}$ ) as described by de Duve and co-workers $(5,19)$. The " $L$ " fraction was

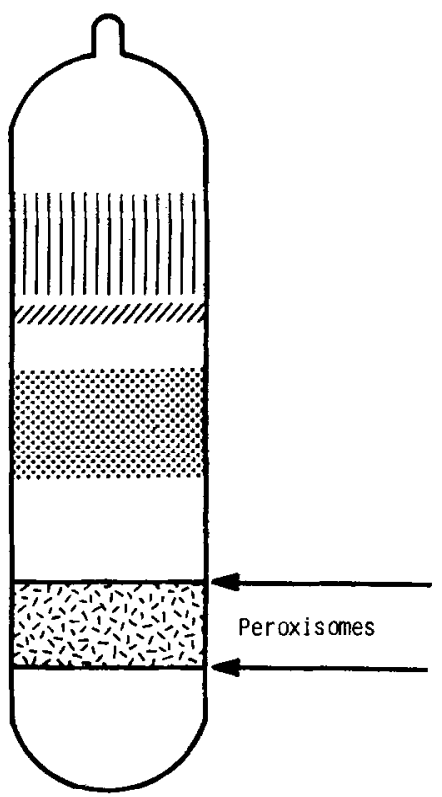

FIG. 1. Schematic representation of the banding patterns of " $L$ " fraction after centrifugation in a 20-50\% Metrizamide density gradient in a vertical rotor. 


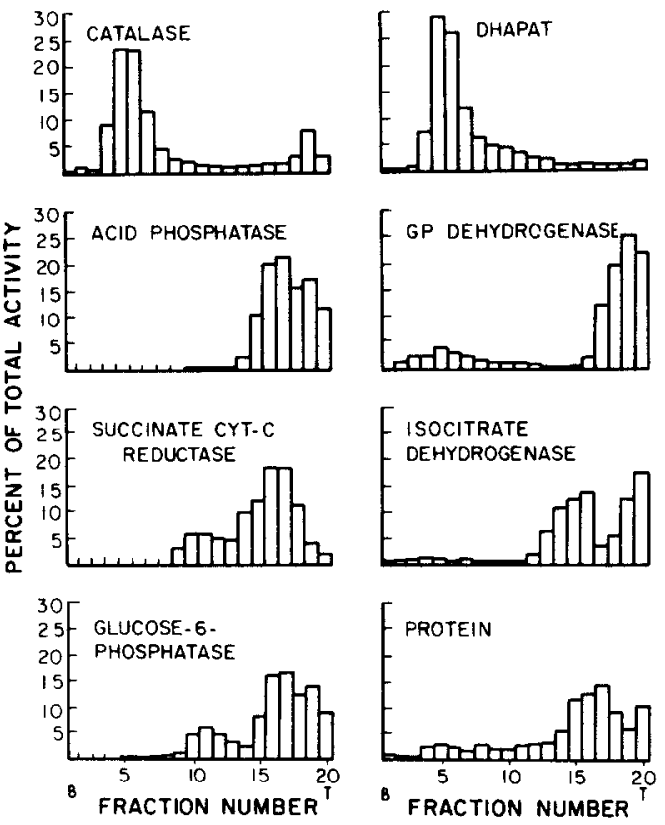

FIG. 2. Distribution pattern of different enzymes of rat liver light mitochondrial (L) fraction (24 $\mathrm{mg}$ protein) after centrifugation in a linear Metrizamide density gradient (20-50\% Metrizamide, density 1.11 to $1.28 \mathrm{~g} /$ $\mathrm{ml}$ ). Following centrifugation in a vertical rotor and fraction collection (see text for details) each fraction was assayed for different subcellular marker enzymes. The recovery of each enzyme was $106 \%$ for catalase (peroxisomal marker enzyme), $92 \%$ for acid phosphatase (lysosomal marker enzyme), $85 \%$ for succinate-cytochrome $c$ reductase (mitochondrial marker enzyme), 94\% for glucose-6-phosphatase (microsomal marker enzyme), $95 \%$ for DHAP acyltransferase (DHAPAT), and $97 \%$ for protein. B, Bottom of gradient (50\% Metrizamide); T, top of the gradient (20\% Metrizamide).

washed twice $(25,000 \mathrm{~g}, 10 \mathrm{~min})$ with the homogenizing buffer (sucrose-Tris-EDTA) and then suspended in the same buffer in such a way that $1 \mathrm{ml}$ of the suspension contained the "L" fraction obtained from 4 $\mathrm{g}$ of tissue.

Metrizamide density gradient centrifugation. The $20-50 \%$ linear Metrizamide gradients were prepared directly in the Beckman Quick Seal centrifuge tubes $(25 \times 89 \mathrm{~mm})$. Six different Metrizamide solutions of increasing densities $(20,26,32,38,44$, and $50 \%$ ) were layered vertically below each other by injecting these solutions $(6.6 \mathrm{ml}$ each of increasing densities for $40-\mathrm{ml}$ tube) to the bottom of the centrifuge tube through a long hollow needle from the top. The narrow openings of the tubes were sealed with Parafilm and the tubes were slowly and gently tilted to the horizontal position and kept at $4^{\circ} \mathrm{C}$ for $15-20 \mathrm{~h}$. Before the loading, the tubes were slowly returned to the vertical positions, and then about 1-2 $\mathrm{ml}$ of Metrizamide solution from the top was removed and replaced with the corresponding volume of S-1 or "L" suspensions with the aid of a Beckman sample application apparatus. Gradients were also sometimes made by a conventional gradient making apparatus (BioRad Model 230) using 20 and 50\% Metrizamide solutions. However, this latter method was found to be more tedious and somewhat less reproducible than the method described above.

After the loading, the tubes were heat sealed using Quick Seal tube sealer (Beckman) and centrifuged in a vertical rotor (Beckman VTi50) using an ultracentrifuge (Beckman L8-70) at a total centrifugal force $\left(\omega^{2} t\right)$ of 6.3 $\times 10^{10}$ radians $^{2} / \mathrm{sec}\left(130,000 g_{\text {av }}\right.$ for $65-70$ min). After centrifugation the tubes were opened by cutting off the seal and the contents were pumped out from the bottom by a peristaltic pump through a long hollow needle which was inserted from the top. Fractions of about $2 \mathrm{ml}$ were collected in a fraction collector and the fractions were quick-frozen in a dry ice-acetone bath and stored at $-20^{\circ} \mathrm{C}$.

Enzyme assays. Established procedures have been used to assay the marker enzymes such as catalase (21), acid phosphatase $(22,23)$, succinate-cytochrome $c$ reductase (24), and glucose-6-phosphatase (25). Uric acid oxidase was determined as described by Leighton et al. (5) after complete removal of Metrizamide by extensive dialysis of the fractions against $0.25 \mathrm{M}$ sucrose. Because of its strong absorption in the uv region, Metrizamide interferes with the uric acid oxidase assay at $292 \mathrm{~nm}$. Monoamine oxidase was assayed using labeled tyramine as described 


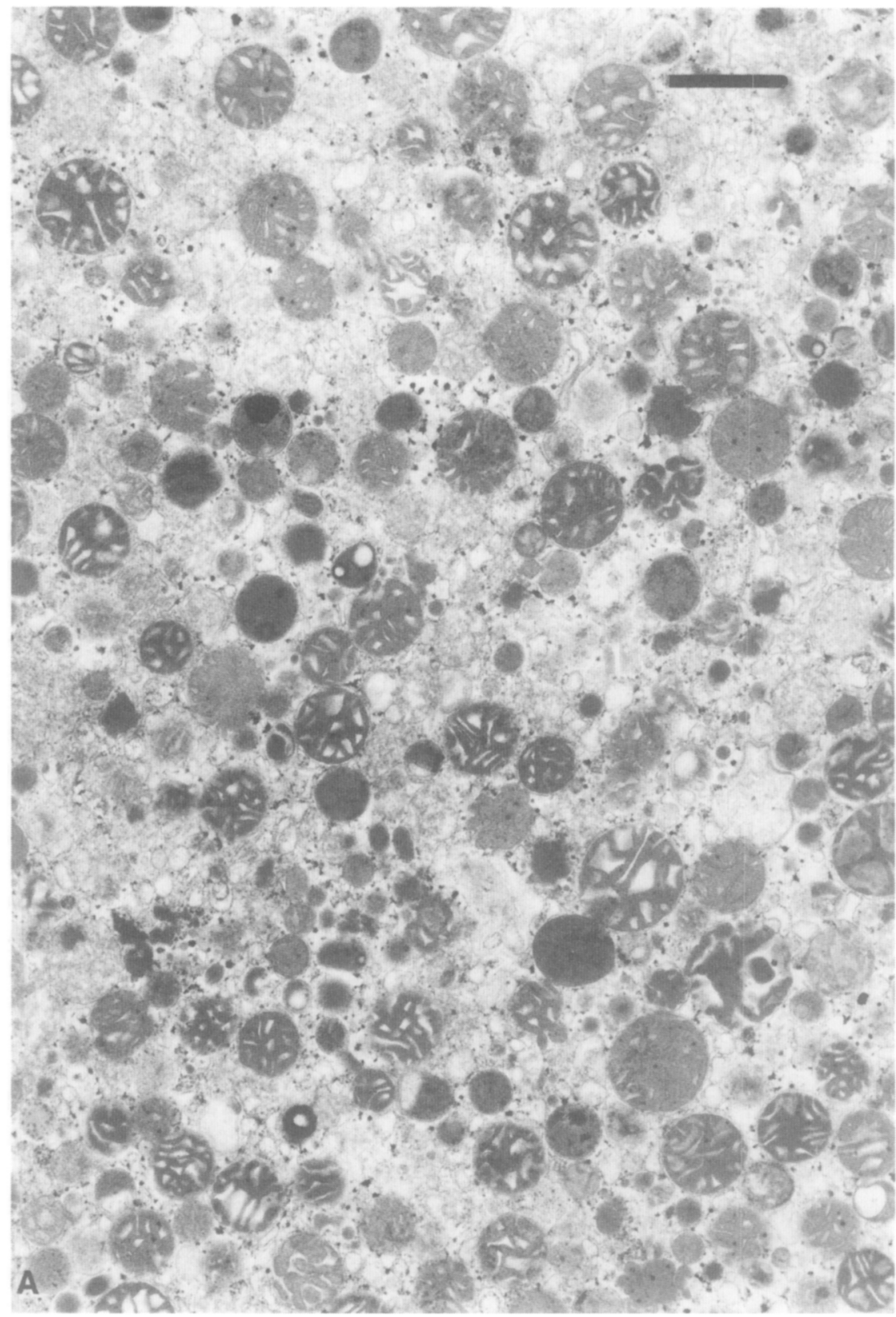

FIG. 3. Morphological appearance of rat liver (A) L fraction and (B) purified peroxisomal fraction when examined under electron microscope (see text for details). Bar $=1 \mu \mathrm{m}$.

by Wurtman and Axelrod (26). DHAP acyltransferase and GP acyltransferase activities were determined by measuring the labeled lipids formed either from $\left[{ }^{32} \mathrm{P}\right] \mathrm{DHAP}$ or from $\left[{ }^{32} \mathrm{P}\right] \mathrm{GP}$ and palmitoyl CoA $(6,17)$. Palmitoyl
CoA synthase activity was measured by the filter paper assay method of Roughan and Slack (27) and palmitoyl CoA hydrolase was assayed as described by Berge (28). Cyanideinsensitive fatty acid $\beta$-oxidation was deter- 


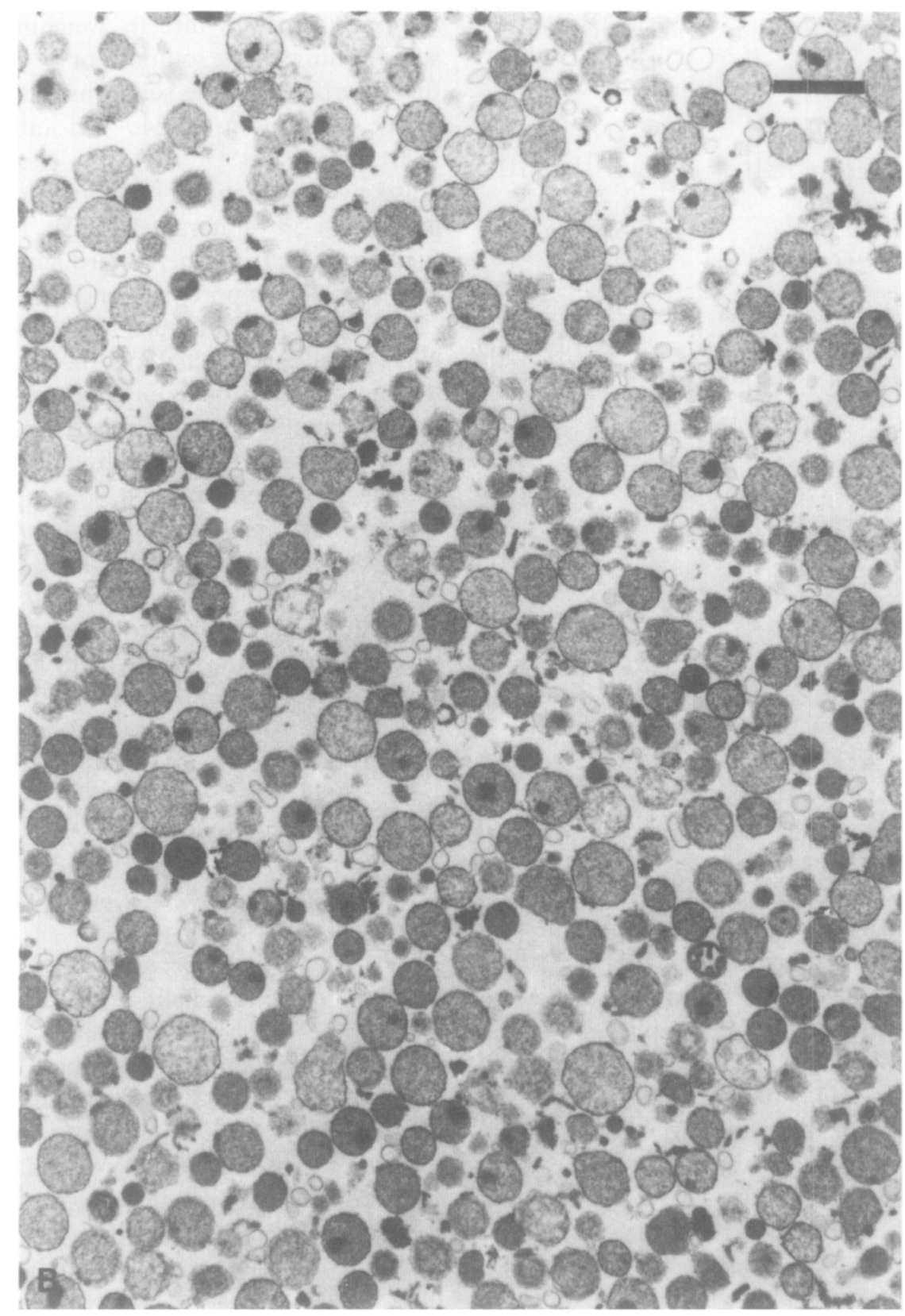

FIG. 3-Continued.

mined by measuring the acid-soluble radioactivity formed from $\left[1-{ }^{14} \mathrm{C}\right]$ palmitoyl $\mathrm{CoA}$ as described by Lazarow (29). Isocitrate dehydrogenase was assayed as described by Kornberg (30).
A radiometric assay was developed to measure low GP dehydrogenase activity in the peroxisomal fractions. The incubation mixture contained Tricine buffer ( $\mathrm{pH} 7.8,50$ $\mathrm{mM}), \mathrm{NADH}(0.3 \mathrm{mM}),\left[{ }^{32}\right.$ P]DHAP $(0.5$ 


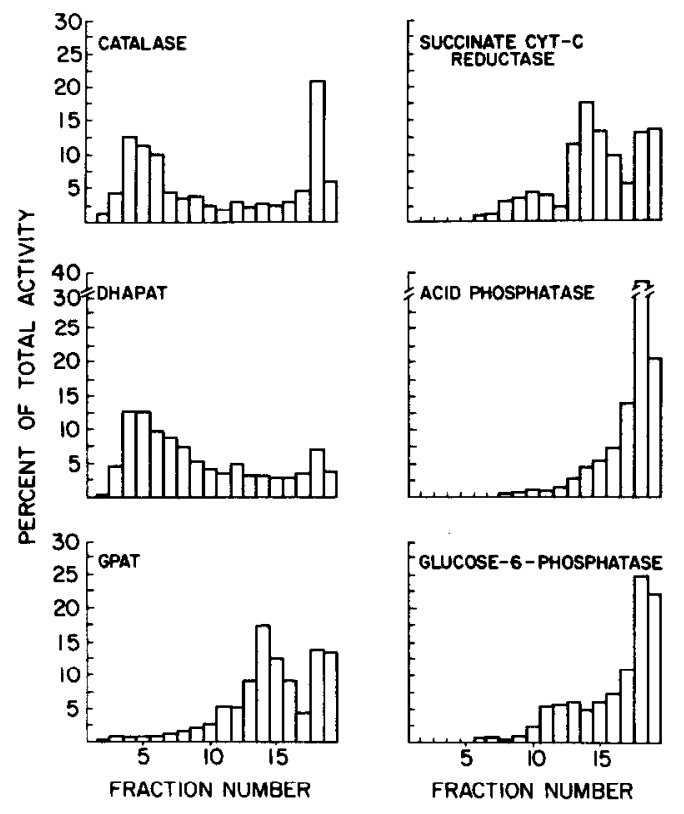

FIG. 4. Distribution pattern of different enzymes of rat kidney " $L$ " fraction after centrifugation in Metrizamide density gradient similar to that described in Fig. 2. GPAT, GP acyltransferase.

$\left.\mathrm{mM}, 8 \times 10^{5} \mathrm{cpm}\right)$, and the fractions $(2-20$ $\mu \mathrm{g}$ protein) in a total volume of $0.1 \mathrm{ml}$. The mixture was incubated at $37^{\circ} \mathrm{C}$ for $5 \mathrm{~min}$ and the reaction was stopped by adding 50 $\mu \mathrm{l}$ of $0.3 \mathrm{M}$ oxalate buffer ( $\mathrm{pH} 1.5$ ). An aliquot $(20 \mu \mathrm{l})$ of the mixture was subjected to high-voltage paper electrophoresis at $\mathrm{pH}$ 1.5 to separate the product $\left[{ }^{32} \mathrm{P}\right] \mathrm{GP}$ from $\left[{ }^{32} \mathrm{P}\right]$ DHAP (31). After separation, the radioactive spots on the paper were localized by radioautography and cut out, and the radioactivities in these spots were quantified by liquid scintillation counting (32). The reaction rate for this assay was found to be linear with time (up to $5 \mathrm{~min}$ ) and protein concentrations (up to $30 \mu \mathrm{g}$ protein).

Other methods. Protein was determined either by the dye-binding method (33) or by a modified Lowry (34) assay in which the protein was first coprecipitated with deoxycholate by trichloroacetic acid. This was done to remove the Metrizamide which interferes with the Lowry assay procedure $(11,33)$. The densities of Metrizamide solutions were de- termined either by directly weighing a measured volume of the solutions or by measuring the absorbance of the solutions (after appropriate dilutions) at $240 \mathrm{~nm}$ (absorbance $=3.12 \times 10^{4} \mathrm{M}^{-1} \mathrm{~cm}^{-1}$ ). For electron microscopy the fractions in Metrizamide were fixed in $1.5 \%$ glutaraldehyde at $4^{\circ} \mathrm{C}$. The fractions were then filtered through Millipore filters $\left(0.025-\mu \mathrm{m}\right.$ pore size) under $\mathrm{N}_{2}$ pressure as described by Baudhuin (22). The subcellular particles collected on the filters were then postfixed in osmium tetroxide solution $\left(1 \% \mathrm{OsO}_{4}\right.$ in $50 \mathrm{mM}$ phosphate buffer at $\mathrm{pH}$ 7.4) for $2 \mathrm{~h}$ and then dehydrated by serially passing the filters through solutions of increasing ethanol concentrations. After the final immersion in $100 \%$ ethanol, the filters are embedded in Spurr resin. Thin sections were cut on an LKB microtome and viewed, after counterstaining lightly with lead citrate and uranyl acetate, in a Philips 400 electron microscope. All other procedures were the same as described previously $(6,17,20)$.

\section{RESULTS}

\section{Isolation of Peroxisomes from Rat Liver " $L$ " Fraction}

After density gradient centrifugation, the peroxisomes from the rat liver " $L$ " fraction form a diffuse band near the bottom of the tube $(\rho=1.22)$ well separated from other organelles (Fig. 1). This is clearly seen in the distribution profile of different marker enzymes in this Metrizamide gradient (Fig. 2). Peroxisomes (marker enzyme catalase) are localized mainly in the bottom half of the tube (fractions 4-7) whereas lysosomes (marker enzyme acid phosphatase), mitochondria (marker enzyme succinate-cytochrome $c$ reductase), and microsomes (marker enzyme glucose-6-phosphatase) are all localized mainly in the top half (above fraction 9) of the tube. A small amount of catalase is also present at the top of the gradient which is probably due to soluble catalase released from the breakdown of a small fraction of 
peroxisomes during handling of the " $L$ " fraction. DHAP acyltransferase, which has been shown to be a peroxisomal enzyme $(6,16,20)$, sediments with the catalase-containing particles (Fig. 2). The enrichment (relative specific activity) of catalase and DHAP acyltransferase in the peak fractions (fractions 5 and 6) starting from the " $L$ " fraction is about 10- and 12-fold, respectively. Uric acid oxidase, another peroxisomal enzyme, is also found to sediment with the catalase-containing particles (data not shown; however, see Fig. 7). A part of cellular glycerophosphate dehydrogenase has been reported to be present in peroxisomes (35). This is confirmed here as shown in Fig. 2. About $16 \%$ of total glycerophosphate dehydrogenase present in the " $L$ " fraction is found to be present in peroxisomes. Similarly, as reported before (5), a small $(6 \%)$ but measurable amount of isocitrate dehydrogenase (NADPH specific) was also found to be present in peroxisomes (Fig. 2).

The Metrizamide from peroxisomes can be removed by diluting the fractions with $0.25 \mathrm{M}$ sucrose and then pelleting the peroxisomes by centrifugation at $100,000 \mathrm{~g}$ for $30 \mathrm{~min}$. A portion of the peroxisomes, however, is damaged by this procedure as seen by the release of $10-20 \%$ catalase in the supernatant. A more gentle procedure for the removal of Metrizamide is to dialyze the fractions against $0.25 \mathrm{M}$ sucrose at $4^{\circ} \mathrm{C}$ for 10-12 h. Peroxisomes isolated by such procedure are intact since it was found that all the catalase is particulate bound and cannot be inactivated by brief trypsin treatment (10 min at $25^{\circ} \mathrm{C}$ ) unless detergent (Triton $\mathrm{X}$ 100 ) is also present.

Morphological examination of the peroxisomal fraction isolated by this Metrizamide gradient centrifugation technique also showed that the peroxisomes are uncontaminated with other subcellular organelles. This is shown in Fig. 3 where it is seen that though the starting " $L$ " fraction contained large numbers of mitochondria, lysosomes, and endoplasmic reticulum residues (Fig. 3A), these organelles are practically absent in the isolated peroxisomal fraction (Fig. 3B).

\section{Isolation of Peroxisomes from Rat Kidney}

Peroxisomes from rat kidney " $L$ " fraction can also be isolated by a similar Metrizamide density gradient centrifugation in a vertical rotor. The results are shown in Fig. 4. As in the rat liver, the peroxisomes (catalase-containing particles) sedimented to the bottom half of the tube away from mitochondria, lysosomes, and microsomes (Fig. 4). Morphometric examination also shows that the isolated peroxisomes are pure (Fig. 5).

\section{Isolation of Peroxisomes from Mouse Liver Postnuclear Supernatant}

The procedure described above can be used to isolate peroxisomes from guinea pig liver " $L "$ fraction (16) and also from the liver postnuclear supernatant in which most of the other subcellular organelles are also present. This is shown by using mouse liver in Figs. 6 and 7. It is seen that when mouse liver postnuclear homogenate is loaded on a linear Metrizamide density gradient and spun in a vertical rotor, the peroxisomes are sedimented as a band close to the bottom of the tube well separated from other organelles. The two marker enzymes for peroxisomes, catalase and urate oxidase, are cosedimented at the same position. However, a large proportion of catalase, compared to that of " $L$ " fraction, is present at the top of the gradient. This may represent soluble and/or microperoxisomal-bound catalase. DHAP acyltransferase measured at both $\mathrm{pH} 5.5$ and $\mathrm{pH} 7.5$ has a distribution pattern very similar to that of catalase (Fig. 7). In contrast GP actyltransferase, which is known to be present in mitochondria and microsomes, is absent from the peroxisomal fraction (Fig. 7). The cyanide-insensitive fatty acid $\beta$-oxidation enzyme system, which is known to be peroxisomal (36), comigrates with sedimentable catalasecontaining particles. The lysosomes (marker 


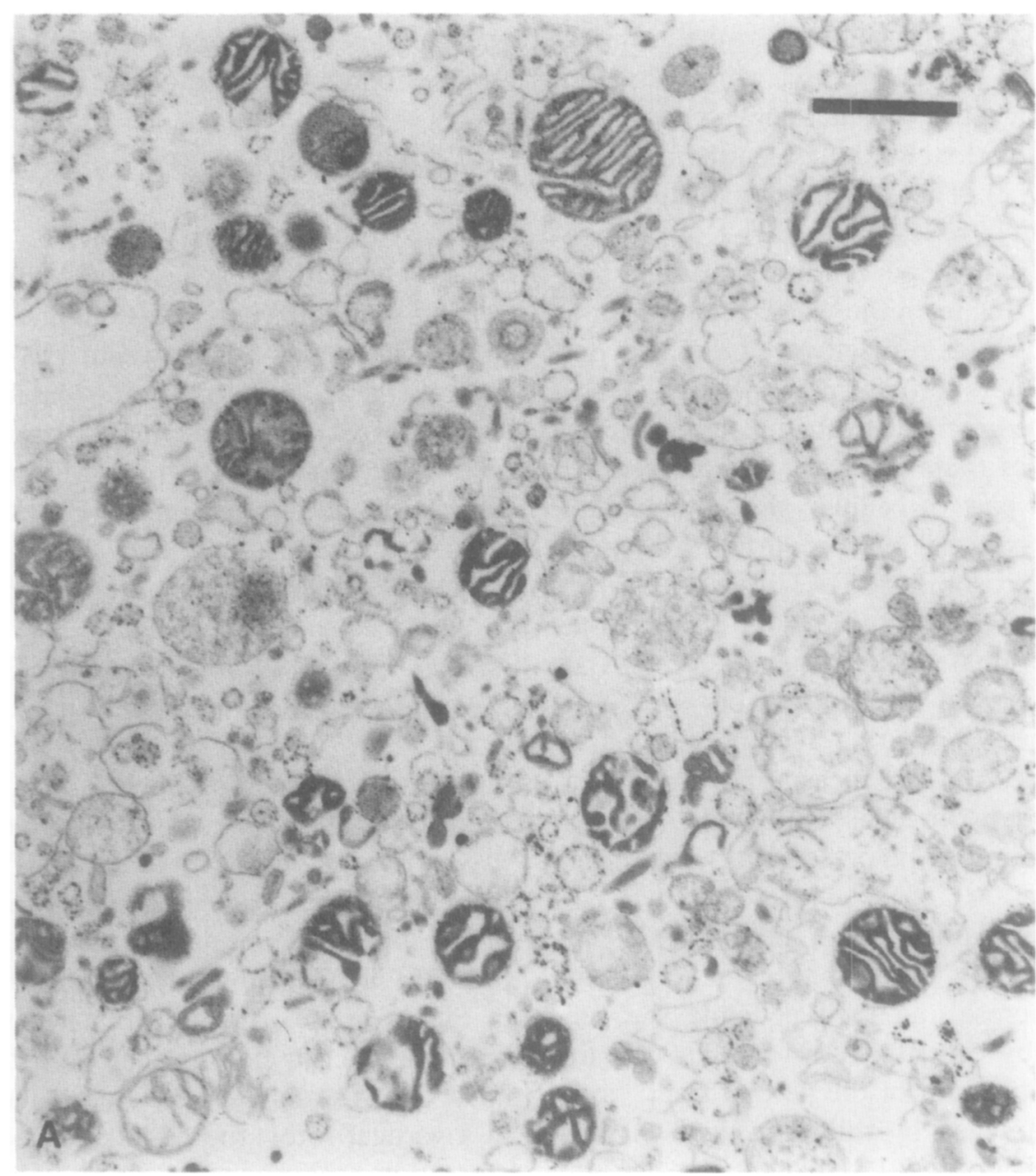

FIG. 5. Morphological appearance of rat kidney (A) L fraction and (B) the combined peroxisomal fractions (see text for details). Bar $=1 \mu \mathrm{m}$.

enzyme acid phosphatase) are clearly separated out from peroxisomes (Fig. 6) but fractions of both the microsomes (marker enzyme glucose-6-phosphatase) and mitochondria (marker enzymes succinate-cytochrome $c$ reductase and monoamine oxidase) migrate close to (but well separated from) the peroxisomal peak. A small fraction (4.0\%) of mitochondrial outer membrane (marker enzyme monoamine oxidase) contaminates the peroxisomal fraction (Fig. 7). The results in Fig. 6 also confirm the presence of palmitoyl CoA ligase in peroxisomes which has been reported by Krisans et al. (37). However, palmitoyl CoA hydrolase, the activity of which has been shown to be increased by peroxisome proliferators (38), is not present in peroxisomes (Fig. 6). In separate experiments (data not shown), the combined peroxisomes fraction was found to be uncontaminated with plasma membrane (marker enzyme 5'-nucleotidase) and golgi bodies (marker enzyme galactosyl transferase). In the peak fractions catalase and DHAP acyltransferase are seen (Fig. 8) to be enriched (i.e., relative specific activity) by $25-$ to 30 fold from S-1 (30- to 35-fold from the wholeliver homogenate). 


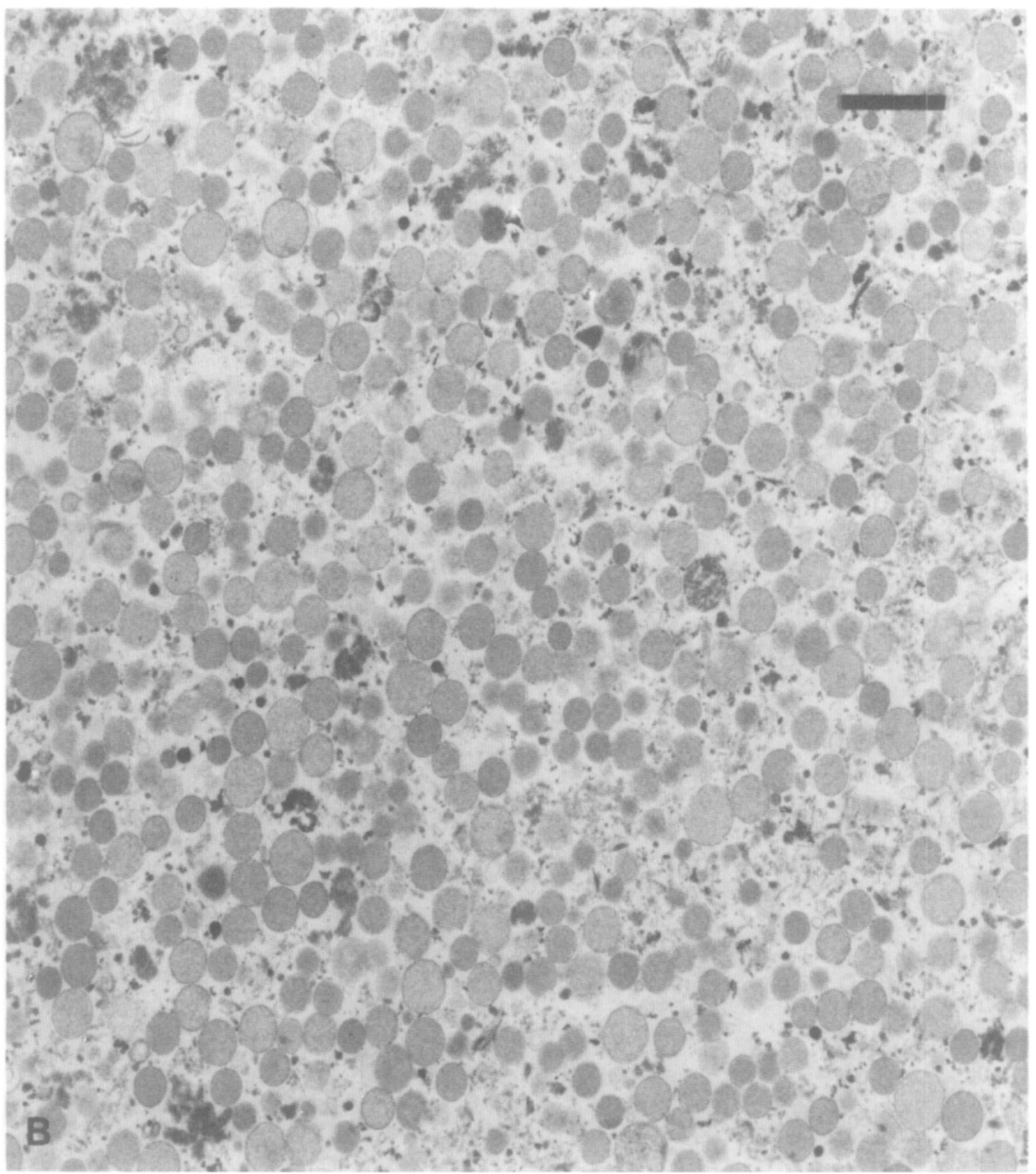

FIG. 5.-Continued.

\section{DISCUSSION}

It is clear from the results presented above that on centrifugation the liver and kidney peroxisomes migrate faster than any other organelle and appear as a distinct band in this Metrizamide density gradient. The enrichment of catalase from rat liver homogenate in the peak peroxisomal fractions is about 40- to 50-fold, which is similar to that reported by other workers (5). From the distribution profiles of the marker enzymes, it is seen that the main peroxisome fractions contain very little contamination from any other subcellular fractions even when the total postnuclear homogenate was used for the separation. Only on the low-density side of the peroxisome band is there some contamination by microsomes due to the presence of the microsomal shoulder peak. However, part of the contamination is probably due to the fraction collection method employed (through a hypodermic needle inserted from the top) which caused some mixing inside the vertical needle and tube due to the inversion of the gradient. Visually, a clear demarcation was seen between the pale brown peroxisomal band and the reddish microsomal band after centrifugation (Fig. 1).

These results show that peroxisomes from 

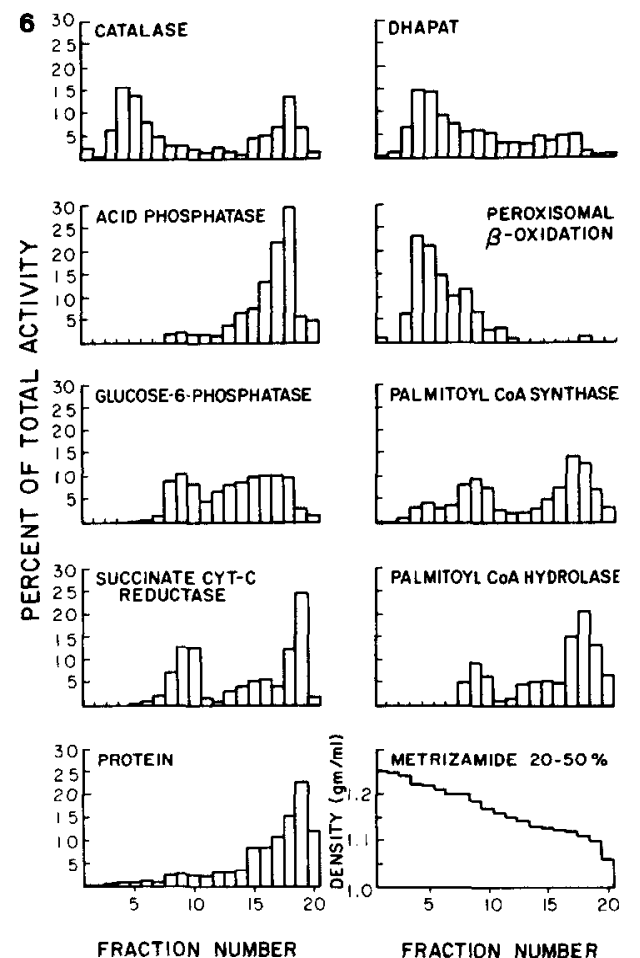
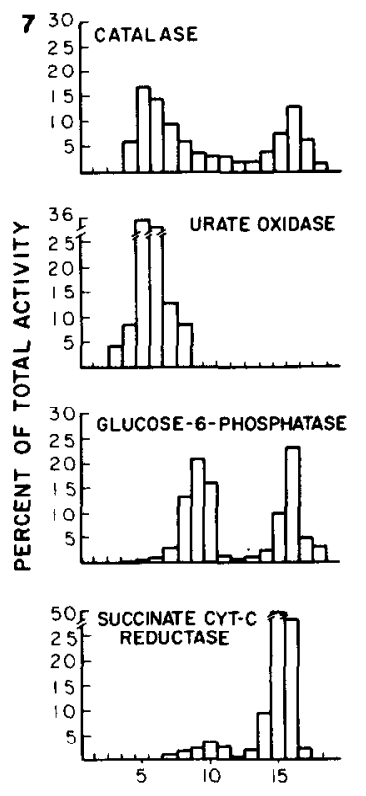

FRACTION NUMBER
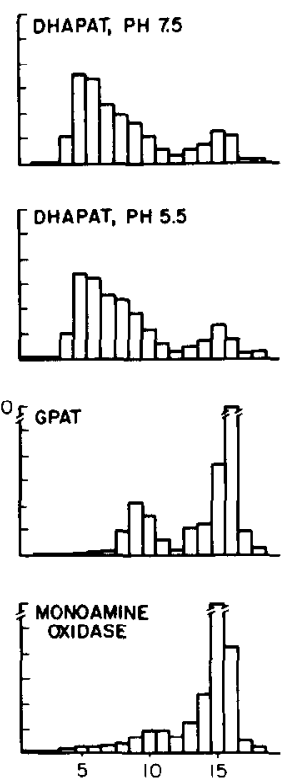

FRACTION NUMBER

FiG. 6 and 7. Distribution patterns of different enzymes in two different preparations of mouse liver S1 fraction (42 and $45 \mathrm{mg}$ protein; see Fig. 2 legend for details). The recovery of the marker enzymes is between $90-105 \%$.

kidney can also be isolated by this method in a relatively pure state. Kidney peroxisomes uncontaminated with other organelles have not been isolated before. It is also seen that, as in liver, DHAP acyltransferase in kidney has a very similar distribution pattern to that of catalase, indicating that this enzyme is exclusively localized in peroxisomes. Therefore, it seems DHAP acyltransferase may be used as a peroxisomal membrane marker enzyme. The similarities of distribution of activity of DHAP acyltransferase when measured at $\mathrm{pH} 5.5$ and 7.5 further indicate that a part of pH 7.5 activity is not due to the nonspecificity of GP acyltransferase. The distribution of GP acyltransferase in the Metrizamide gradient fractions is quite different from that of DHAP acyltransferase (Figs. 4 and 7).

As mentioned before, Metrizamide density gradient centrifugation has been used by a number of authors for the preparative isolation of peroxisomes from liver. Wattiaux et al. (11) first demonstrated that lysosomes can be separated from peroxisomes in Metrizamide density gradient centrifugation. Bronfman et al., using isopycnic Metrizamide density gradient centrifugation in a swingingbucket rotor, isolated peroxisomes from liver (12). Similar methods have been used by Small et al. (39) and Crane et al. (40) to isolate peroxisomes from liver and kidney. In all these methods the isolated peroxisomes are contaminated with varying amounts of microsomes and other organelles. This is probably because of long periods of centrifugation employed at high hydrostatic pressure for the isopycnic operation. The peroxisomes separate from lysosomes and mitochondria in the Metrizamide medium probably because peroxisomes, but not lysosomes or mitochondria, are permeable to Metrizamide. In 


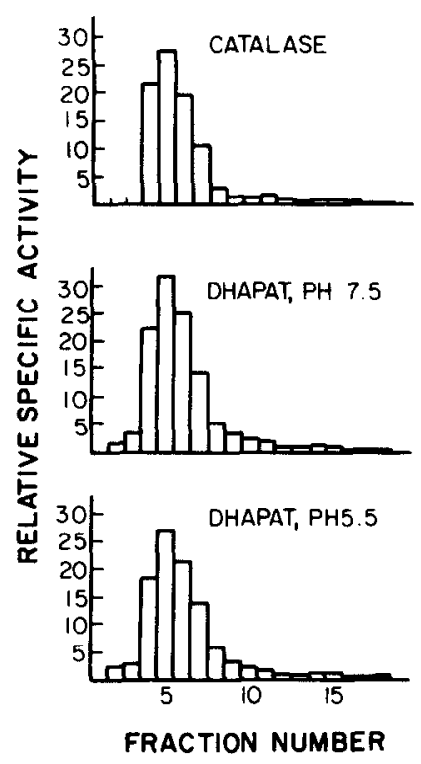

FIG. 8. The enrichment of the mouse liver peroxisomal enzymes after Metrizamide density gradient centrifugation. The enrichment is calculated as the relative specific activities of these enzymes in these fractions compared to those of the starting S-1 fraction.

other words, as defined by de Duve and coworkers, the Metrizamide space of peroxisomes is much larger than that of lysosomes or mitochondria $(41,42)$. Therefore, during centrifugation in the vertical rotor, due to the short path length, peroxisomes sediment to their intrinsic equilibrium density more quickly than do lysosomes and mitochondria. The microsomal vesicles because of their smaller size sediment at a slower rate, i.e., rate-dependent banding as defined by Reid and Williamson (43), than do peroxisomes. Longer centrifugation times than employed here results in more microsomal contamination of the peroxisome fractions.

The question naturally arises whether similar Metrizamide density gradient centrifugation can be employed to isolate microperoxisomes from other tissues. Our preliminary results using developing rat brain indicate that, though it is possible to enrich the microperoxisomes from brain homogenate using such gradients, the microperoxisomes cannot be isolated completely free of microsomes
(K. O. Webber and A. K. Hajra, unpublished results). This is probably due to the similarity in sizes of microperoxisomes and endoplasmic reticulum vesicles.

Sucrose and Percoll density gradient centrifugation have also been used to isolate peroxisomes from liver $(5,8-10,13-15)$. The peroxisomes isolated in Percoll gradients are contaminated with other organelles, especially with mitochondria. Also, it is difficult to separate the insoluble Percoll (colloidal silica particles) from the subcellular organelles. Similarly, peroxisomes free from lysosomes can be isolated by sucrose density gradient centrifugation only when the rats are pretreated by injecting Triton WR-1339 to lower the density of lysosomes. A special rotor (Beaufay rotor) is also needed to purify peroxisomes by this method (10). Metrizamide is superior to sucrose because of its lower osmolarity at the same density of the solutions $(11,42)$. Also, in a vertical rotor the hydrostatic pressure during the centrifugation is much lower than that exerted in swingingbucket rotors (13). From the results presented here, it is evident that Metrizamide density gradient centrifugation in a vertical rotor is most suited to isolation of pure peroxisomes from normal liver and kidney in a relatively short period of time. The only disadvantage of this procedure is the high cost of Metrizamide. However, recently a compound, $\mathrm{Ny}$ codenz (5-[N-2,3dihydroxypropyl acetamido)2,4,6-tri-iodo- $N$, $N^{\prime}$-bis[2,3dihydroxypropyl]isophthalamide), similar in structure to $\mathrm{Me}$ trizamide, has been developed (Nyegaard \& Co.) which is somewhat less expensive. Preliminary experiments in this laboratory (A. K. Hajra and D. Wu, unpublished results) and in other laboratories ${ }^{3}(44)$ indicate that Nycodenz, instead of Metrizamide, may be used for the isolation of peroxisomes.

\footnotetext{
${ }^{3}$ Peroxisomes isolated by the method described by Osmundsen (44) is contaminated with microsomes. This is probably due to the step gradient used for the centrifugal separation of peroxisomes. We found that by using a linear gradient, as described above, the peroxisomes are uncontaminated with microsomes.
} 


\section{ACKNOWLEDGMENTS}

This work is supported by research Grants NS 15747 and NS 08841 from the National Institutes of Health. The expert technical assistance of $\mathrm{Mr}$. James Schreiber for the electron microscopy is gratefully acknowledged.

\section{REFERENCES}

1. de Duve, C., and Baudhuin, P. (1966) Physiol. Rev. 46, 323-357.

2. de Duve, C. (1969) Proc. Roy. Soc. London. Ser. B 173, $71-83$.

3. Novikoff, A. B., and Goldfischer, S. (1969) J. Histochem. Cytochem. 17, 675-680.

4. Novikoff, A. B., Novikoff, P. M., Quintana, N., and Davis, C. (1972) J. Histochem. Cytochem. 20, 747-749.

5. Leighton, F., Poole, B., Beaufay, H., Baudhuin, P., Coffey, J. W., Fowler, S., and de Duve, C. (1968) J. Cell. Biol. 37, 482-513.

6. Hajra, A. K., Burke, C. L., and Jones, C. L. (1979). J. Biol. Chem. 254, 10896-10900.

7. Ishii, H., Suga, T., Hayashi, H., and Ninobe, S. (1979) Biochim Biophys. Acta. 582, 213-220.

8. Tolbert, N. E. (1976) in Methods in Enzymology (Fleischer, S., and Packer, L., eds.), Vol. 31, Part A, pp. 734-746, Academic Press, New York.

9. Connock, M. J., and Temple, N. J. (1983) Int. J. Biochem. 15, 125-131.

10. Fujiki, Y., Fowler, S., Shio, H., Hubbard, A. L., and Lazarow, P. B. (1982) J. Cell. Biol. 93, 103-110.

11. Wattiaux, R., Wattiaux-de Connick, S., RonveauxDupal, M., and Dubois, F. (1978) J. Cell. Biol. 78, 349-368.

12. Bronfman, $M$., Inestrosa, N. C., and Leighton, F. (1979) Biochem. Biophys. Res. Commun. 88, 1030-1036.

13. Neat, C. E., Thomassen, M. S., and Osmundsen, $H$. (1980) Biochem. J. 186, 369-371.

14. Appelkvist, E. L., Brunk, U., and Dallner, G. (1981) J. Biochem. Biophys. Methods 5, 203-217.

15. Mannaerts, G. P., Van Veldhoven, P., Van Broekhoven, A., Vandebroek, G., and Debeer, T. J. (1982) Biochem. J. 204, 17-23.

16. Hajra, A. K., and Bishop, J. E. (1982) Ann. N. Y. Acad. Sci. 386, 170-182.

17. Hajra, A. K., and Burke, C. (1978) J. Neurochem. 31, $125-134$.

18. Bishop, J. E., and Hajra, A. K. (1980) Anal. Biochem. 106, 344-350.

19. de Duve, C., Pressman, B. C., Gianetto, R., Wattiaux, R., and Appelmans, F. (1955) Biochem. J. 60, 604-617.

20. Jones, C. L., and Hajra, A. K. (1977) Biochem. Biophys. Res. Commun. 76, 1138-1143.

21. Peters, T. J., Muller, M., and de Duve, C. (1972) J. Exp. Med. 136, 1117-1139.
22. Baudhuin, P. (1974) in Methods in Enzymology (Fleischer, S., and Packer, L., eds.), Vol. 31, Part A, pp. 356-368, Academic Press, New York.

23. Chen, P. S., Jr., Toribara, T. Y., and Warner, H. (1956) Anal. Chem. 28, 1756-1758.

24. Schnaitman, C., and Greenawalt, J. W. (1968) J. Cell. Biol. 38, 158-175.

25. Nordlie, R. C., and Arion, W. J. (1966) in Methods in Enzymology (Wood, W. A., ed.), Vol. 9, pp. 619-625, Academic Press, New York.

26. Wurtman, R. J., and Axelrod, J. (1964) Biochem. Pharmacol. 12, 1439-1440.

27. Roughan, P. G., and Slack, C. R. (1977) Biochem. J. 162, 457-459.

28. Berge, R. K. (1979) Biochim. Biophys. Acta 574, $321-333$.

29. Lazarow, P. B. (1981) in Methods in Enzymology (Lowenstein, J. M., ed.), Vol. 72, Part D, pp. 315-319, Academic Press, New York.

30. Kornberg, A. (1955) in Methods in Enzymology (Colowick, S. P., and Kaplan, N. O., eds.), Vol. 1, pp. 705-707, Academic Press, New York.

31. Hajra, A. K., and Agranoff, B. W. (1968) J. Biol. Chem. 243, 1617-1622.

32. LaBelle, E. F., and Hajra, A. K. (1972) J. Biol. Chem. 247, 5825-5834.

33. Gogstad, G. O. (1980) Anal. Biochem. 106, 524528.

34. Bensadoun, A., and Weinstein, D. (1976) Anal. Biochem. 70, 241-250.

35. Gee, R., McGroarty, E., Hsieh, B., Wied, D. M., and Tolbert, N. E. (1974) Arch. Biochem. Biophys. 161, 187-193.

36. Lazarow, P. B. (1978) J. Biol. Chem. 253, 15221528.

37. Krisans, S. K., Mortensen, R. M., and Lazarow, P. B. (1980) J. Biol. Chem. 255, 9599-9607.

38. Kawashima, Y., Katoh, H., and Kozuka, H. (1982) Biochim. Biophys. Acta. 712, 48-56.

39. Small, G. M., Hocking, T. J., Sturdee, A. P., Burdett, K., and Connock, M. J. (1981) Life Sci. 28, 1875-1882.

40. Crane, D., Holmes, R., and Masters, C. J. (1982) Biochim. Biophys. Acta. 712, 57-64.

41. Beaufay, H., Jacques, P., Baudhuin, P., Sellinger, O. Z., Berthet, J., and de Duve, C. (1964) Biochem. J. 92, 184-205.

42. Wattiaux, R., and Wattiaux-de Coninck (1983) in Iodinated Density Gradient Media (D. Rickwood, ed.), pp. 119-138, IRL Press, Oxford/Washington, D. C.

43. Reid, E., and Williamson, R. (1974) in Methods in Enzymology (Fleischer, S., and Packer, L., eds.), Vol. 31, Part A, pp. 713-733, Academic Press, New York.

44. Osmundsen, H. (1983) in Iodinated Density Gradient Media (D. Rickwood, ed.), pp. 139-146, IRL Press, Oxford/Washington, D. C. 